\title{
SIMRES-TV: NOISE AND RESIDUAL SIMILARITY FOR PARAMETER ESTIMATION IN TOTAL VARIATION
}

\author{
V. B. Surya Prasath ${ }^{1,2,3,4}$, Nguyen Ngoc Hien $^{5}$, Dang N. H. Thanh ${ }^{6, *}$, Sergey Dvoenko ${ }^{7}$ \\ ${ }^{1}$ Division of Biomedical Informatics, Cincinnati Children's Hospital Medical Center, Cincinnati, OH 45229 USA \\ ${ }^{2}$ Department of Pediatrics, University of Cincinnati College of Medicine, Cincinnati, OH 45257, USA \\ ${ }^{3}$ Department of Biomedical Informatics, College of Medicine, University of Cincinnati, OH 45267 USA \\ ${ }^{4}$ Department of Electrical Engineering and Computer Science, \\ University of Cincinnati, OH 45221 USA - prasatsa@uc.edu \\ 5 Dong Thap University, Cao Lanh City, Vietnam - nguyenngochien@ dthu.edu.vn \\ ${ }^{6}$ Department of Information Technology, School of Business Information Technology, \\ University of Economics Ho Chi Minh City, Vietnam - thanhdnh@ueh.edu.vn \\ ${ }^{7}$ Institute of Applied Mathematics and Computer Science, Tula State University, Russia - dvsrge@ gmail.com
}

\section{Commission II/5, WG II/10}

KEY WORDS: Image Restoration, Regularization, Total Variation, Parameter Estimation, Residual Similarity, Convex Minimization

\begin{abstract}
:
Image restoration with regularization models is very popular in the image processing literature. Total variation (TV) is one of the important edge preserving regularization models used, however, to obtain optimal restoration results the regularization parameter needs to be set appropriately. We propose here a new parameter estimation approach for total variation based image restoration. By utilizing known noise levels we compute the regularization parameter by reducing the similarity between residual and noise variances. We use the split Bregman algorithm for the total variation along with this automatic parameter estimation step to obtain a very fast restoration scheme. Experimental results indicate the proposed parameter estimation obtained better denoised images and videos in terms of PSNR and SSIM measures and the computational overload is less compared with other approaches.
\end{abstract}

\section{INTRODUCTION}

Total variation (TV) regularization is introduced by Rudin et al (Rudin et al., 1992) for obtaining edge preserving restorations of noisy images. Due to its edge preserving property the TV model is used widely in image processing and other related areas (Prasath et al., 2015). If we let $f: \Omega \subset \ell^{2} \rightarrow[0,255]$ be the (noisy) input image, $|\Omega|=m \times n$ is the image domain and size. Solving the following unconstrained minimization provides an estimate of the latent image $f=u+\epsilon$ with additive Gaussian noise $\epsilon$ of standard deviation $\sigma$ (assumed known),

$$
\min _{u} \mathcal{E}_{\lambda}(u)=\lambda T V(u)+\sum_{1 \leq i, j \leq M, N}\left(u_{i j}-f_{i j}\right)^{2},
$$

where $T V(u)=\sum_{1 \leq i, j \leq m, n}\left|(\nabla u)_{i j}\right|$ is the discrete total variation with $(\nabla u)_{i j}=\left(u_{i+1, j}-u_{i j}, u_{i, j+1}-u_{i j}\right)^{T}$, with zero boundary conditions. The regularization parameter $\lambda>0$ is crucial in obtaining meaningful results and traditionally it is left as a tunable parameter (Prasath et al., 2017, Prasath et al., 2018). Larger $\lambda$ values result in over-smoothing whereas smaller $\lambda$ parameter value results in noise being kept, see Figure 1. As can be seen from the residual images (Fig. 1(middle row)) more structures are removed as we increase the $\lambda$ value. Thus, a systematic approach in selecting this crucial regularization parameter is paramount in obtaining accurate denoising results. There exists various methods for choosing the regularization parameter such as the Lagrange multiplier (Rudin et al., 1992), discrepancy principle, L-curve, cross-validation (Hansen, 2010)

\footnotetext{
* Corresponding author
}

generalized cross validation (Reeves, Mersereau, 1990) etc. Fixing a parameter with these standard parameter estimation methods can still lead to poor results, since the regularization needs to be reduced in the iterative implementation. Recently data adaptive methods (Fu, Zhang, 2010, Prasath, Singh, 2010, Wang et al., 2011, Prasath, 2011, Prasath, Moreno, 2018) are found to provide better solutions, though with higher computational overhead.

If we assume that the noise level is known (or an upper bound), then we can utilize it to tune $\lambda$ dynamically through the iterations. This means that we solve (1) for both the image and $\lambda$, which is a bi-objective optimization (Weiss et al., 2009, Beck, Teboulle, 2009). In this work, our main aim is to use a similarity criteria based parameter estimation for TV minimization model with a fast implementation. Among a wide variety of iterative methods available to solve the TV minimization (Vogel, Oman, 1998, Dobson, Vogel, 1997, Blomgren et al., 1997, Chambolle, 2004, Osher et al., 2005), recently the split Bregman (Goldstein, Osher, 2009) is proven to be very fast and used widely in the literature. By using the fact that in TV based denoising with an iterative algorithm the similarity between the residual and noise variance (SIMRES) should approach zero, we devise an algorithm that can help select the regularization parameter $\lambda$ effectively. We test the SIMRES-TV on a variety noisy images and compare with other regularization parameter selection models (Rudin et al., 1992, Hansen, 2010, Reeves, Mersereau, 1990, Prasath, Singh, 2010). Our experimental results indicate that SIMRES-TV can obtain better results with comparable or exceeding image quality in denoising natural images. Further, the proposed SIMRES approach, apart from with TV, can also 

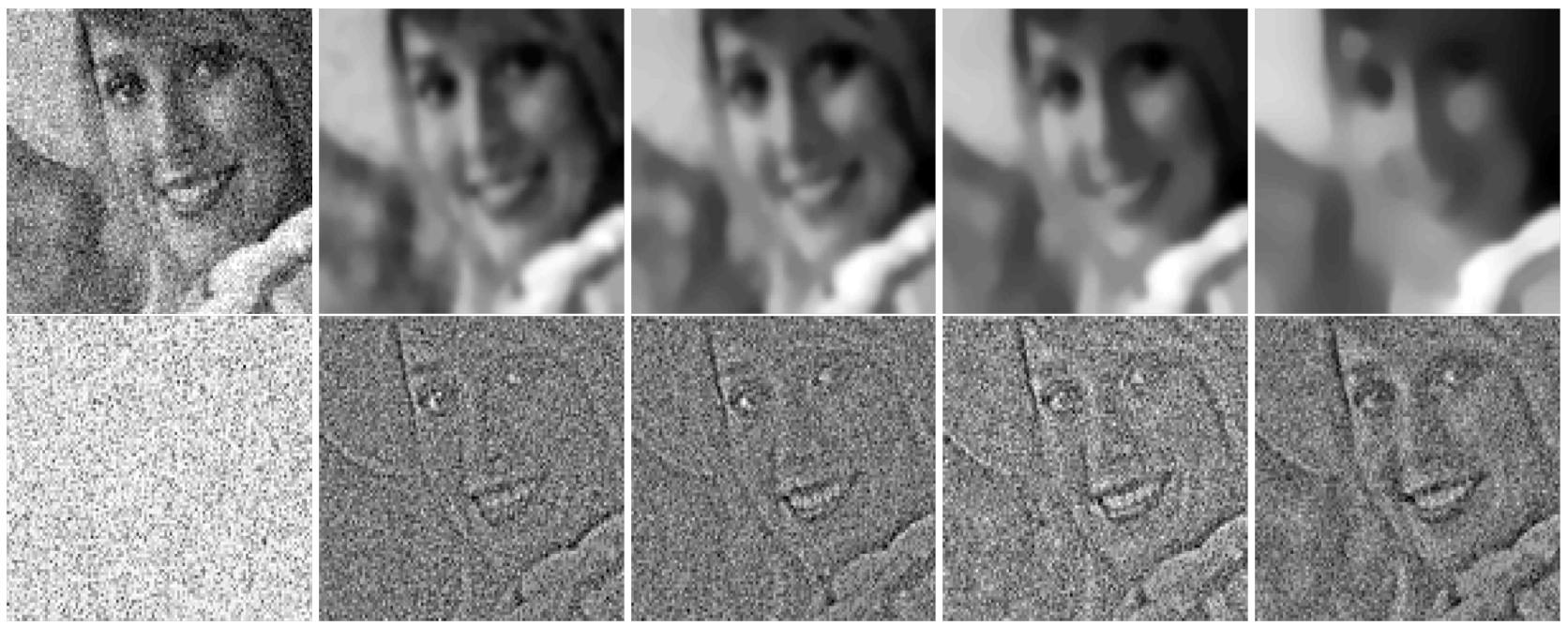

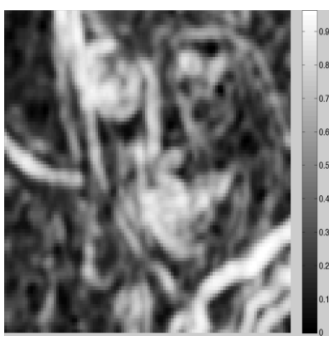

(a) Noisy

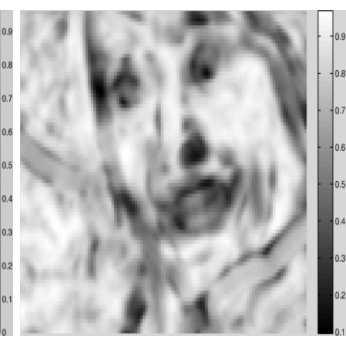

(b) 0.1

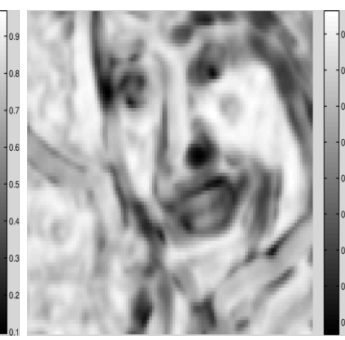

(c) 0.5

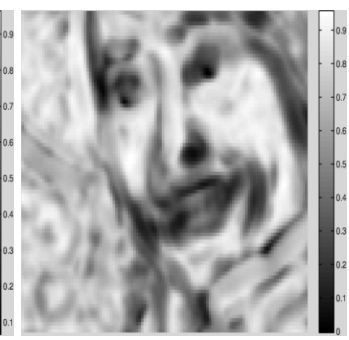

(d) 0.75

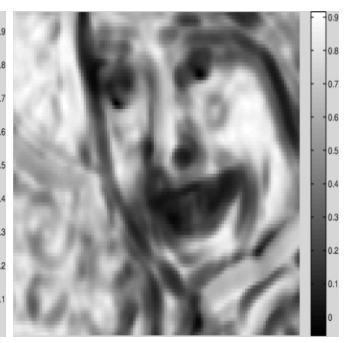

(e) 1

Figure 1. Top row: TV regularization (1) based image restoration results for different constant $\lambda$ values. Middle row: Noise amount (shown in (a) with gray values reversed for better visualization), and corresponding residual images $|u-f|$. Bottom row: Structural similarity (SSIM) maps between original image and resultant images. Larger $\lambda$ blurs edges and converges to a piecewise constant image.

be augmented to any convex regularization functions with convergence guarantees.

Our paper is organized as follows. Section 2 introduces the SIMRES-TV algorithm. Section 3 provides the experimental details and comparison of denoising various natural images. Finally, Section 4 concludes the paper.

\section{PARAMETER ESTIMATION WITH SIMRES CRITERIA}

We first note that the similarity between residual and noise variances (SIMRES) can be quantified with the following equation:

$$
R(u)=\frac{\left|(u-f)^{2} / M N-\sigma^{2}\right|}{\sigma^{2}}
$$

Ideally, in image denoising, we require that $R(u) \rightarrow 0$, however in typical iterative optimization schemes, it can take a large number of iterations to achieve such requirement, and usually constraining the residue closer to zero, $R(u)<T h$ with $T h$ small, would suffice to obtain meaningful restorations. Imposing such a constraint on $R(u)$ along with minimizing a regularization such as the TV (1) can be undertaken and that leads to selecting appropriate regularization parameter $\lambda$. We thus use a decision based on the smaller SIMRES criterion for determining the $\lambda$ parameter and use it to minimize the TV objective functional (1). To motivate this, we present an example of denoising a natural image corrupted with Gaussian noise in Figure 1 with different $\lambda$ values based TV regularization. As can be seen, setting $\lambda$ high removes finer details and obtains piecewise constant results. In contrast, using smaller $\lambda$ values fails to obtain cleaner results. Instead of hand-tuning the parameter, in this work, we embed decreasing $\lambda$ values which were tuned by the relative residual term into the minimization of (1). The overall algorithm is as follows:

1. Initialise $\lambda^{1}, T h$, and $u^{0}=f$.

(a) We use mean filtered ( $3 \times 3$ averaging kernel) image $\bar{f}$ of input $f$ to compute the initial $\lambda^{1}=\xi^{0} \frac{\left(f_{i j}-\bar{f}_{i j}\right)^{2}}{T V(\bar{f})}$ with initial value $\xi^{0}=0.5$.

(b) Compute a minimiser of Eqn. (1),

$$
u^{1}=\arg \min _{v} \mathcal{E}_{\lambda^{1}}(v)
$$

2. For $k=2, \ldots$ do:

(a) Reduce the parameter value using the following sequence,

$$
\lambda^{k}=\xi^{k-1} \frac{\sum_{i j}\left(u_{i j}^{k-1}-f_{i j}\right)^{2}}{T V\left(u_{i j}^{k-1}\right)}
$$

where $\xi^{k-1}>0$ is a parameter.

(b) Compute a minimiser of Eqn. (1), 

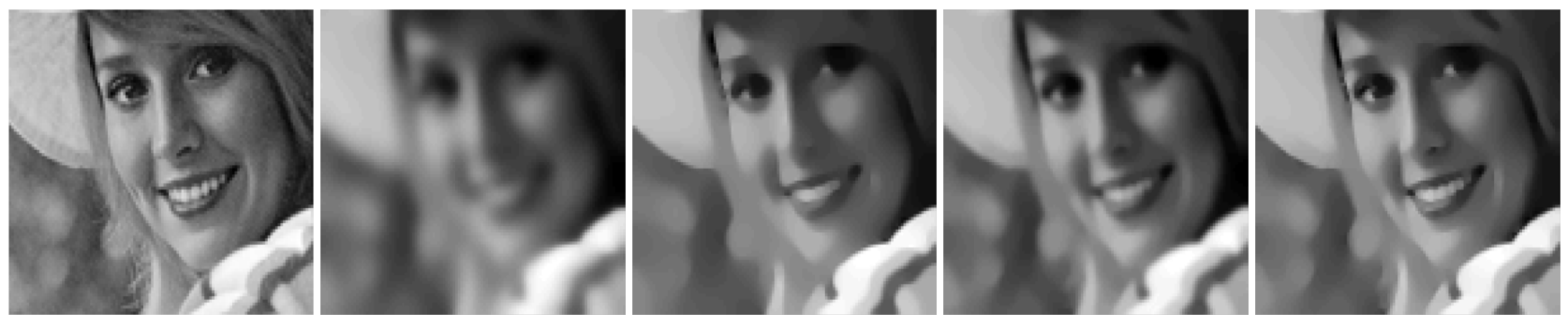

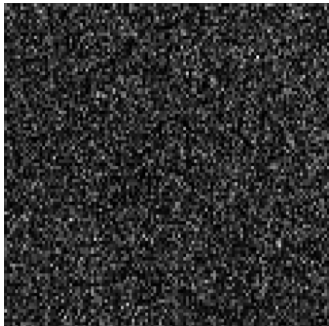

(a) Noise-free

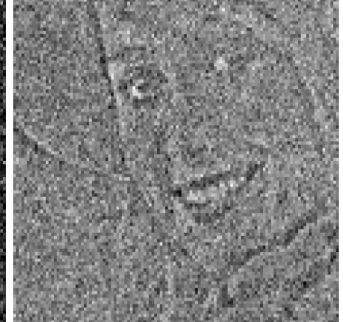

(b) LM

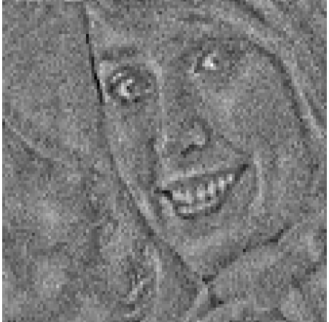

(c) GCV

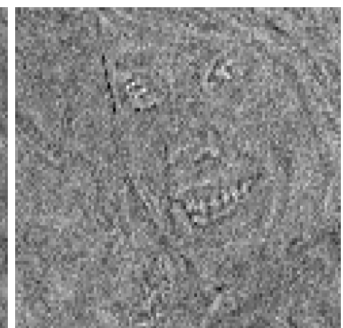

(d) IG

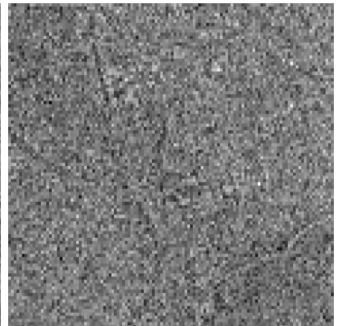

(e) SIMRES

Figure 2. Comparison of different $\lambda$ selection methods in image restoration with TV minimization. Top row: Restoration results. Bottom row: Residue. (a) Noise-free, and restoration by (b) LM, (c) GCV, (d) IG, (e) our SIMRES-TV. In the (a) bottom figure we show the amount of noise that need to be removed. Better viewed online and zoomed in.

$$
u^{k}=\arg \min _{v} \mathcal{E}_{\lambda^{k}}(v)
$$

3. Stop if either $R\left(u^{k}\right)<T h$ or $\left|u^{k-1}-u^{k}\right|<T o l$.

We use the split Bregman algorithm (Goldstein, Osher, 2009) to solve the discrete energy minimizations at Steps 1, and 2(b). That is, we used the following steps:

$$
\begin{aligned}
\left(u^{k+1}, \vec{d}^{k+1}\right) & =\arg \min _{0 \leq \phi \leq 1, \vec{d}}\left|\vec{d}_{g}\right|+\frac{\lambda}{2}\left\|\vec{d}-\nabla u-\vec{b}_{L^{2}(\Omega)}^{2}\right\| \\
\vec{b}^{k+1} & =\vec{b}^{k}+\nabla u^{k}-\vec{d}^{k}
\end{aligned}
$$

where $\vec{d}$ is computed with a shrinkage operator

$$
\vec{d}^{k+1}=\operatorname{shrink}\left(\nabla u^{k}+\vec{b}^{k}, 1 / \lambda\right),
$$

where $\operatorname{shrink}(x, \gamma)=x /|x| * \max (|x|-\gamma, 0)$. We refer to work of (Goldstein, Osher, 2009) for more details of the original split Bregman formulation and variants.

The scaling parameter is chosen to be decreasing, i.e., $\xi^{k}=$ $\xi^{k-1} / \gamma$ and this guarantees the convergence of our algorithm to a minimal solution of (1). Note that we only compute an approximate solution (local-minima) of the energy functional (1) at these steps. Since the TV regularization based minimization is convex, we are guaranteed to find a minima and the solution corresponds to an optimal denoising result in our case. The Perona-Malik type models (Perona, Malik, 1990) correspond to non-convex regularization functions and thus convergence can not guaranteed under the SIMRES algorithm.

\section{EXPERIMENTAL RESULTS AND COMPARISONS}

All the images and variables are normalized to $[0,1]$ range, and noise level $\sigma^{2}=0.01$. The threshold $T h=0.1$, scaling parameter $\gamma=10$ and tolerance $T o l=10^{-12}$ are fixed. Tolerance based stopping criteria is used for other schemes reported
Table 1. PSNR(dB)/MSSIM values for different automatic parameter based TV regularization restoration results for noisy Elaine image. Last row shows the computation time in seconds.

\begin{tabular}{|l|c|c|}
\hline Model & PSNR/MSSIM & Time \\
\hline Noisy & $19.39 / 0.3936$ & - \\
LM (Rudin et al., 1992) & $23.03 / 0.6313$ & 10 \\
GCV (Reeves, Mersereau, 1990) & $23.38 / 0.7333$ & 12 \\
IG (Prasath, Singh, 2010) & $21.32 / 0.7648$ & 18 \\
SIMRES-TV (Ours) & $27.25 / 0.8245$ & 13 \\
\hline
\end{tabular}

here. We compare with the Lagrange multiplier (LM) (Rudin et al., 1992), inverse gradient (IG) (Prasath, Singh, 2010, Prasath, Moreno, 2018, Thanh et al., 2020), generalized cross validation (GCV) (Reeves, Mersereau, 1990) along with TV minimization.

Figure 2 shows restoration of Elaine grayscale image for different parameter estimation methods. We show in 2(a) top row the original noise-free image, and bottom row the amount of noise that need to be removed. Residual images clearly show that SIMRES based restoration is better in terms of edge preservation and does not remove edge pixels as observed in the other results. In LM (Rudin et al., 1992) based result we see uniform blurring, whereas in GCV (Reeves, Mersereau, 1990) piecewise constant regions can be observed. IG (Prasath, Singh, 2010) provided better results than LM, and GCV but removed some edges as can be seen on the corresponding residue image. In contrast, the proposed SIMRES-TV model obtained better result without removing edges, compare the random noise in (a) with the residue image in (e).

For quantitative measurements we utilize the peak signal to noise ratio - PSNR (in decibels, $\mathrm{dB}$ ) and the structural similarity - SSIM (range $[0,1]$ ) image quality metrics. Table 1 shows the PSNR, mean SSIM values and computation time (in seconds) corresponding to restorations shown in Figure 2. Apart from achieving better quality values, the SIMRES-TV based implementation is faster as well as computationally efficient. In terms of edge preservation, SSIM value indicate that the structural details are kept well by the SIMRES-TV with more than $6 \mathrm{~dB}$ increase in PSNR values over the nearest model (Prasath, Singh, 


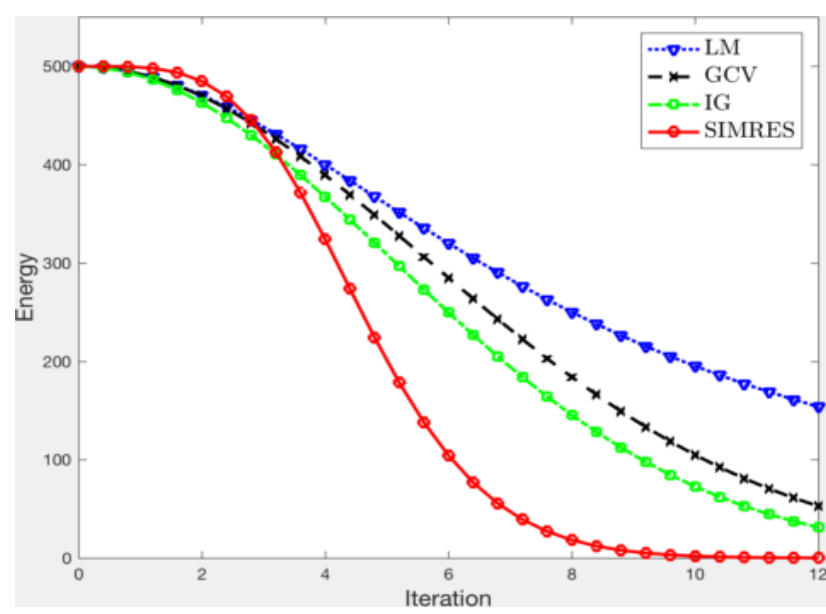

Figure 3. Energy Vs iteration for different parameter selection methods with Elaine image.

Table 2. Comparison of different methods on a standard test images from the USC-SIPI dataset with Gaussian noise $\sigma_{n}=20$. We show the mean SSIM values with best results are in boldface.

\begin{tabular}{l|cccc}
\hline Images & LM & GCV & IG & $\begin{array}{c}\text { Our } \\
\text { SIMRES }\end{array}$ \\
\hline Couple & 0.7503 & 0.7353 & 0.7298 & $\mathbf{0 . 8 2 2 4}$ \\
F-16 & 0.8128 & 0.8432 & 0.71099 & $\mathbf{0 . 8 6 8 1}$ \\
Girl1 & 0.7930 & 0.8027 & 0.8291 & $\mathbf{0 . 8 8 2 2}$ \\
Girl2 & 0.8934 & 0.8729 & 0.823 & $\mathbf{0 . 9 0 1 1}$ \\
Girl3 & 0.7825 & 0.8006 & 0.8178 & $\mathbf{0 . 8 4 4 1}$ \\
House & 0.7299 & 0.7545 & 0.8128 & $\mathbf{0 . 8 5 1 3}$ \\
IPI & 0.8841 & 0.839 & 0.8745 & $\mathbf{0 . 9 2 0 4}$ \\
IPIC & 0.8924 & 0.8764 & 0.8925 & $\mathbf{0 . 9 2 3 8}$ \\
Tree & 0.7335 & 0.7395 & 0.704 & $\mathbf{0 . 8 1 2 9}$ \\
Baboon & 0.5687 & 0.4597 & 0.4701 & $\mathbf{0 . 6 0 3 2}$ \\
Barbara & 0.6892 & 0.7624 & 0.7131 & $\mathbf{0 . 8 6 1 7}$ \\
Boat & 0.6973 & 0.6419 & 0.6078 & $\mathbf{0 . 7 8 3 9}$ \\
Car & 0.7891 & 0.7543 & 0.677 & $\mathbf{0 . 8 4 3 1}$ \\
Lena & 0.753 & 0.8563 & 0.8443 & $\mathbf{0 . 8 9 9 7}$ \\
Peppers & 0.8942 & 0.8567 & 0.8513 & $\mathbf{0 . 9 1 0 5}$ \\
Splash & 0.8967 & 0.9136 & 0.8876 & $\mathbf{0 . 9 2 7 1}$ \\
Tiffany & 0.7596 & 0.7509 & 0.7248 & $\mathbf{0 . 8 4 7 8}$ \\
\hline
\end{tabular}

2010).

Figure 3 shows the energy value $\left(\mathcal{E}_{\lambda}(\cdot)\right.$, see Eqn. (1)) versus iterations for different choices of $\lambda$ parameter estimation and as can be seen SIMRES converges faster than the other schemes with fewer iterations of the split Bregman based implementation.

Next, we show in Table 2 SSIM values for various methods compared with our proposed SIMRES-TV in different standard test images taken from the USC-SIPI Miscellaneous dataset. The input images are corrupted with Gaussian noise of variance $\sigma_{n}=20$. We can see that our SIMRES approach consistently obtains the highest SSIM values among different methods indicating that edges are preserved well across various natural images.

Finally, we provide an example restoration of our SIMRES-TV using a color RGB image Tulip of size $200 \times 200 \times 3$. Figure 4 shows a comparison of restoration results of the methods based on the color-TV (Blomgren, Chan, 1998, Bresson, Chan, 2008) for Gaussian noise of variances of $\sigma_{n}=30,50$ added in each channel. Compared to the traditional color-TV model with either lower $\lambda=0.1$ (noise retainment) or higher
Table 3. Comparison of different quality and error metrics for the Tulip color image with TV regularization for two different noise levels. Best results are in boldface.

\begin{tabular}{l|cccc}
\hline & Noisy & TV & TV & Our \\
& $\sigma_{n}=30$ & $\lambda=0.1$ & $\lambda=1$ & SIMRES \\
\hline ISNR & 0 & 3.2618 & 8.3385 & $\mathbf{9 . 6 9 6 2}$ \\
SNR & 13.4829 & 21.8214 & 23.1791 & $\mathbf{2 6 . 2 8 7 1}$ \\
PSNR & 18.5706 & 26.9091 & 28.2668 & $\mathbf{3 1 . 3 7 4 8}$ \\
\hline MSE & 903.6861 & 132.4865 & 96.9168 & $\mathbf{4 7 . 3 8 0 4}$ \\
RMSE & 30.0614 & 11.5103 & 9.8446 & $\mathbf{6 . 8 8 3 3}$ \\
MAE & 23.9732 & 8.859 & 7.3348 & $\mathbf{5 . 1 6 7 7}$ \\
MAX & 132.8668 & 75.5255 & 91.096 & $\mathbf{8 0 . 6 6 1 4}$ \\
\hline \hline & Noisy & TV & TV & Our \\
& $\sigma_{n}=50$ & $\lambda=0.1$ & $\lambda=1$ & SIMRES \\
\hline ISNR & 0 & 6.6068 & 6.7098 & $\mathbf{1 0 . 3 5 7 2}$ \\
SNR & 9.046 & 15.6527 & 19.4032 & $\mathbf{2 3 . 7 1 4 6}$ \\
PSNR & 14.1337 & 20.7404 & 24.4909 & $\mathbf{2 8 . 8 0 2 3}$ \\
\hline MSE & 2510.2392 & 548.3248 & 231.2018 & $\mathbf{8 5 . 6 7 5 2}$ \\
RMSE & 50.1023 & 23.4163 & 15.2053 & $\mathbf{9 . 2 5 6 1}$ \\
MAE & 39.9553 & 18.2235 & 11.6915 & $\mathbf{6 . 9 2 3 4}$ \\
MAX & 221.4447 & 125.7949 & 109.3267 & $\mathbf{9 6 . 1 6 4 8}$ \\
\hline & & & &
\end{tabular}

$\lambda=1$ (piecewise smoothed) our SIMRES obtains better restored result (devoid of staircasing) by automatically estimating the regularization parameter. We also notice that the residual image in SIMRES does not contain outlines of the tulip and the petals compared to TV regularization with constant regularization parameters. At the higher noise level $\sigma_{n}=50$ traditional TV models retained noise and created staircasing artifacts in flat regions in contrast to smoother SIMRES-TV result. Table 3 shows a comparison of various signal to noise ratio (SNR) image quality metrics (higher is better) and error metrics (lower is better): improved SNR (ISNR, dB), SNR (dB), PSNR (dB), mean squared error (MSE), root MSE (RMSE), maximum absolute error (MAE), maximum absolute error (MAX) for the Tulip color image restorations with two different noise levels $\sigma_{n}=30,50$. Overall, the SIMRES-TV result quantitatively outperformed the standard TV regularization with fixed regularization parameter, obtaining higher SNR based metric values and lower in error metrics.

\section{CONCLUSION}

In this work, we studied a noise and residual similarity measure based parameter estimation for TV regularization scheme in image restoration. The proposed SIMRES approach for estimating the regularization parameter automatically as part of the TV minimization is general in the sense that it can be used along with other (possibly) non-convex minimizations. It will also be interesting to add an independent noise estimation step which will make the proposed method completely automatic for various image processing tasks. Also, combining with other regularization models that utilize adaptive regularization (Prasath, Thanh, 2019) or higher order TV mdoels (Thanh et al., 2020) are also an interesting direction of research.

\section{ACKNOWLEDGEMENTS}

This work was funded by the University of Economics Ho Chi Minh City, Vietnam.

\section{REFERENCES}

Beck, A., Teboulle, M., 2009. Fast Gradient-Based Algorithms for Constrained Total Variation Image Denoising and 


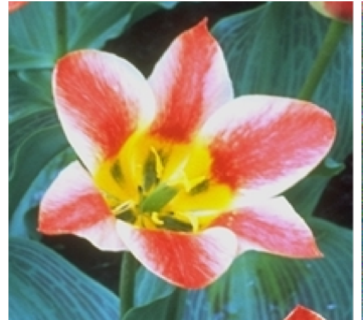

(a) Original

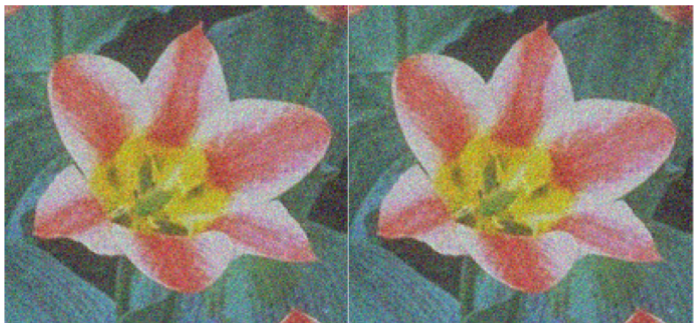

(b) Noisy images $\sigma_{n}=30$, and $\sigma_{n}=50$

TV with $\lambda=0.1$

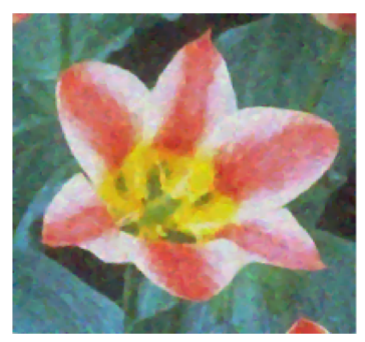

(c) $\sigma_{n}=30$

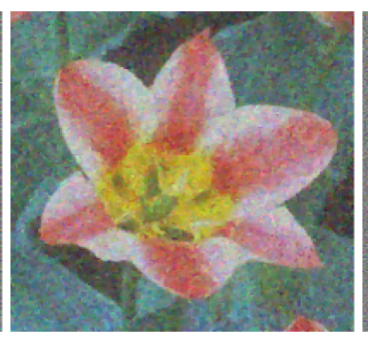

(d) $\sigma_{n}=50$

TV with $\lambda=1$

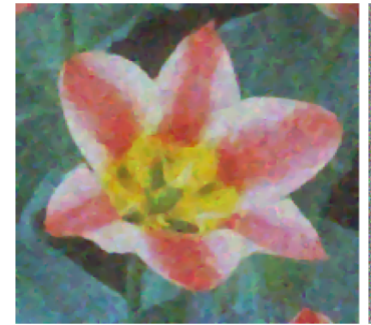

(e) $\sigma_{n}=30$

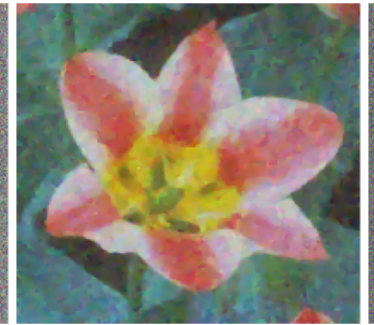

(f) $\sigma_{n}=50$
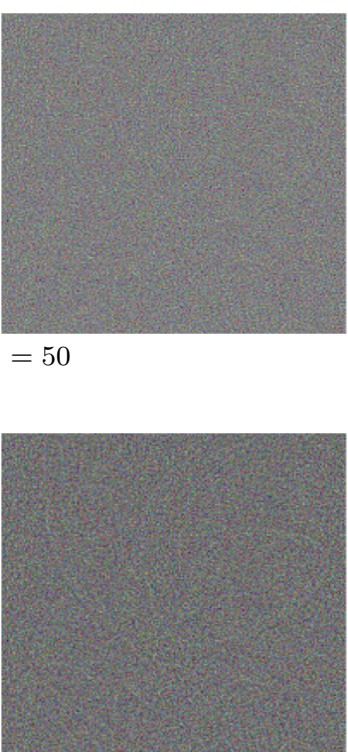

Our SIMRES-TV
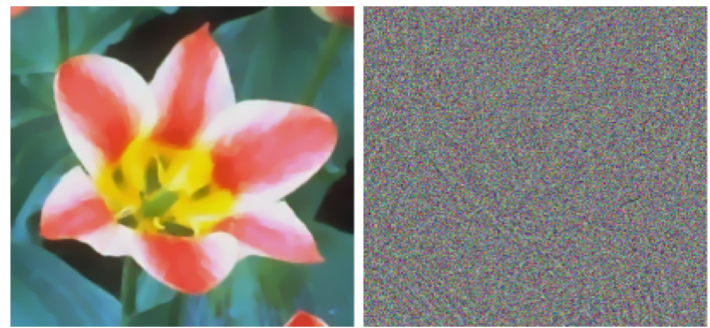

(g) $\sigma_{n}=30$
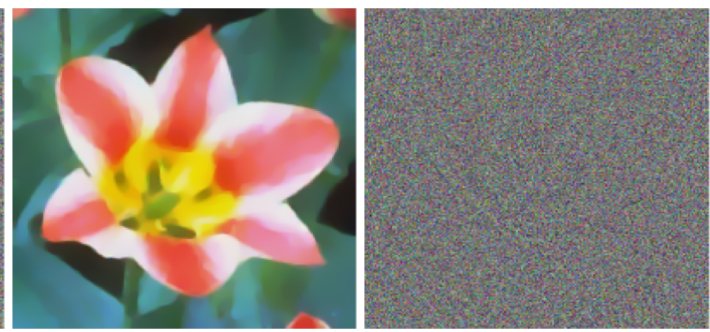

(h) $\sigma_{n}=50$

Figure 4. Color image restoration with TV regularization with constant regularization parameter and SIMRES approach. (a) Original and (b) noisy images (with Gaussian noise variances $\sigma_{n}=30$ and $\sigma_{n}=50$ ). Restoration results with TV regularization parameter (c-d) $\lambda=0.1$, (e-f) $\lambda=1$, and (g-h) our SIMRES-TV. In each of the restoration results, we show restored images on the left and corresponding residual images on the right. 
Deblurring Problems. IEEE Transactions on Image Processing, 18(18), 2419-2434.

Blomgren, P., Chan, T. F., 1998. Color TV: Total variation methods for restoration of vector valued images. IEEE Transactions on Image Processing, 7(3), 304-309.

Blomgren, P., Chan, T. F., Mulet, P., Wang, C. K., 1997. Total variation image restoration: numerical methods and extensions. IEEE International Conference on Image Processing (ICIP), Santa Barbara, CA, USA, 384-387.

Bresson, X., Chan, T. F., 2008. Fast dual minimization of the vectorial total variation norm and applications to color image processing. Inverse Problems and Imaging, 2(4), 455-484.

Chambolle, A., 2004. An Algorithm for Total Variation Minimization and Applications. Journal of Mathematical Imaging and Vision, 20(1-2), 89-97.

Dobson, D. C., Vogel, C. R., 1997. Convergence of an iterative method for total variation denoising. SIAM Journal on Numerical Analysis, 34(5), 1779-1791.

Fu, S., Zhang, C., 2010. Adaptive non-convex total variation regularisation for image restoration. Electronics letters, 46(13), 907-908.

Goldstein, T., Osher, S., 2009. The split Bregman method for L1-regularized problems. SIAM journal on imaging sciences, 2(2), 323-343.

Hansen, P. C., 2010. Discrete inverse problems: insight and algorithms. SIAM, Philadelphia, PA, USA.

Osher, S., Burger, M., Goldfarb, D., Xu, J., Yin, W., 2005. An iterative regularization method for total variation based image restoration. Multiscale Modelling and Simulation, 4(2), 460489.

Perona, P., Malik, J., 1990. Scale-space and edge detection using anisotropic diffusion. IEEE Transactions on Pattern Analysis and Machine Intelligence, 12(7), 629-639.

Prasath, V. B. S., 2011. A Well-posed Multiscale Regularization Scheme for Digital Image Denoising. International Journal of Applied Mathematics and Computer Science, 21(4), 769-777.

Prasath, V. B. S., Moreno, J. C., 2018. On Convergent Finite Difference Schemes for Variational - PDE Based Image Processing. Computational and Applied Mathematics, 37(2), $1562-1580$.

Prasath, V. B. S., Thanh, D. N. H., 2019. Structure tensor adaptive total variation for image restoration. Turkish Journal of Electrical Engineering and Computer Sciences, 27(2), 11471156.
Prasath, V. B. S., Thanh, D. N. H., Hai, N. H., 2018. Regularization parameter selection in image restoration with inverse gradient: Single scale or multiscale? 7th International Conference on Communications and Electronics (ICCE), Hue, Vietnam.

Prasath, V. B. S., Thanh, D. N. H., Hai, N. H., Cuong, N. X., 2017. Image restoration with total variation and iterative regularization parameter estimation. International Symposium on Information and Communication Technology (SoICT), Nha Trang, Vietnam, 378-384.

Prasath, V. B. S., Vorotnikov, D., Pelapur, R., Jose, S., Seetharaman, G., Palaniappan, K., 2015. Multiscale Tikhonov-total variation image restoration using spatially varying edge coherence exponent. IEEE Transactions on Image Processing, 24(12), 5220-5235.

Prasath, V. S., Singh, A., 2010. A hybrid convex variational model for image restoration. Applied Mathematics and Computation, 215(10), 3655-3664.

Reeves, S. J., Mersereau, R. M., 1990. Optimal estimation of the regularization parameter and stabilizing functional for regularized image restoration. Optical Engineering, 29(5), 446-455.

Rudin, L., Osher, S., Fatemi, E., 1992. Nonlinear total variation based noise removal algorithms. Physica D, 60(1-4), 259-268.

Thanh, D. N., Prasath, V. S., Dvoenko, S. et al., 2020. An adaptive method for image restoration based on high-order total variation and inverse gradient. Signal, Image and Video Processing, 1-9.

Vogel, C., Oman, M., 1998. Iterative methods for total variation denoising. SIAM Journal on Scientific Computing, 17(1), 227238 .

Wang, Y., Chen, W., Zhou, S., Yu, T., Zhang, Y., 2011. MTV: modified total variation model for image noise removal. Electronics Letters, 47(10), 592-594.

Weiss, P., Blanc-Feraud, L., Aubert, G., 2009. Efficient schemes for total variation minimization under constraints in image processing. SIAM Journal of Scientific Computing, 31(3), 2047-2080. 